\title{
CURSO DE INGRESO EN QUÍMICA A LA UNIVERSIDAD: UN ESPACIO DE REVISIÓN Y APRENDIZAJE CONCEPTUAL
}

\author{
RAVIOLO, A. Y ANDRADE GAMBOA, J. \\ Centro Regional Universitario Bariloche. Universidad Nacional del Comahue. \\ San Carlos de Bariloche. 8400. Argentina.
}

\section{SUMMARY}

In this article we report a didactic experience, based on a contructivist approach we carried forward in a chemistry classroom in the University Admittance Course, with the aim to promote conceptual revision and learning. Essentially two basic conceptual cores (discontinue nature of matter and conservations of matter properties) were taken into account. We started from previous ideas and pupils' difficulties. Several methodological strategies to overcome them were developed.

\section{INTRODUCCIÓN}

Generalmente los cursos de ingreso a la universidad, en el caso de la Argentina, tienen características restrictivas. La forma más extendida en que esta función selectiva de alumnos se realiza es mediante la presentación y evaluación de extensas guías de problemas.

Estos problemas requieren de los alumnos una resolución basada en estrategias deductivas y matemáticas, que se apoyan en el uso de algoritmos y aplicación mecánica de fórmulas y en donde se ponen en práctica habilidades como incluir datos en ecuaciones en una especie de «rompecabezas». Se le otorga poco lugar a las ideas previas de los alumnos y al aprendizaje conceptual de los temas tratados.

Otro de los aspectos más comunes, que se observa en la enseñanza de la química es el abuso de los «juegos» de química (Shayer y Adey 1981), largos períodos de tiempo en los cuales se aplican juegos de complicadas reglas para resolver los ejercicios; el ejemplo típico es la formuiación de compuestos inorgánicos.

Además de esta caracterización metodológica de la puesta en práctica de los cursos de ingreso, resalta el carácter implícito de obstáculo que ha ser vencido, más que un espacio de aprendizaje que suscite interés y oportunidad de revisión de los conocimientos que poseen los alum- nos sobre los puntos conceptuales centrales de la química.

Es reconocido que las ideas previas condicionan los aprendizajes posteriores (Driver 1986) y, al no ser consideradas, lo que se pretende enseñar se instala como una «capa de barniz» dejando intactas las concepciones erróneas anteriores. Por lo que este tipo de cursos de ingreso ha reforzado un aprendizaje mecánico y memorístico en vez de favorecer aprendizajes significativos (Ausubel, Novak y Hanesian 1978).

En nuestra experiencia llevada a cabo a comienzo de los años 1992 y 1993 , intentamos revertir la situación antes expresada y apoyamos en teorías constructivistas para desarrollar la puesta didáctica.

Teriendo en cuenta contribuciones actuales a la enseñanza de las ciencias (Pozo, Crespo, Sanz y Limón 1992) sobre estructuras cognitivas de grado intermedio entre los estadios generales formulados por Piaget y las ideas específicas del alumno en química, nuestro trabajo, en los últimos años, se inscribe en una aproximación de puesta en aula de los tres núcleos conceptuales propuestos por estos autores. Estos núcleos son: naturaleza discontinua de la materia, conservaciones de propiedades no observables y cuantificación de relaciones. Por 
este ưltimo, se entiende la representación y aplicación cuantitativa de leyes físico-químicas. Es notable cómo, antes de conocer esta línea de investigación, la lógica de la problemática nos llevó a trabajar en una dirección coincidente.

Se presentan a continuación los puntos centrales del plan de trabajo llevado a cabo para facilitar el aprendizaje conceptual y la concepción y fundamentación didáctica en que nos basamos.

\section{SELECCIÓN DE CONTENIDOS}

Dada la corta duración del curso (dos meses de seis horas semanales) y el carácter nivelatorio y de revisión conceptual pretendido, se decidió partir de un conjunto de temas básicos, tratando de presentar una estructura organizada de contenidos.

Las seis unidades formuladas fueron:

1) Materia

2) Compuestos

3) Soluciones

4) Reacciones químicas

5) Estequiometría

6) Estequiometria con soluciones.

Se planificó una secuencia de enseñanza que tuviera en cuenta una presentación gradual en la dificultad de los contenidos, de forma tal que Ios alumnos reafirmen y apliquen las nociones anteriores en cada nueva unidad. Dos ejes conceptuales e integradores que recorren las unidades son: la materia constituida por partículas y las conservaciones de la masa y de los elementos en distintos contextos, enfoque que será profundizado más adelante.

La secuencia de los temas fue pensada de la siguiente manera:

- La materia, sus propiedades y cantidades, como conocimiento básico para elaboración de una teoría corpuscular que explique fenómenos cotidianos y de interés acadérnico.

- Los compuestos como formas de manifestarse la materia (no como producto de una reacción química en esta instancia).

- Las soluciones como una mezcla homogénea de compuestos donde se aplican conceptos básicos de la materia. (naturaleza particular, conservaciones, etc.).

- Las reacciones químicas como transformación de compuestos en otros a través de la ruptura y formación de moléculas, donde se conservan los elementos.
- La estequiometría como relaciones cuantitativas entre los compuestos que participan en una reacción química.

- La estequiometría con soluciones como relaciones cuantitativas entre compuestos que se presentan en solución y reaccionan con otros.

A su vez, hacia el interior de cada unidad didáctica también se procuró una organización de acuerdo a un grado de dificultad y complejidad creciente.

\section{LAS IDEAS DE LOS ALUMNOS}

Se enumeran a continuación algunas dificultades, confusiones típicas y concepciones erróneas de los alumnos en el dominio de fenomenos considerados. En trabajos anteriores (Raviolo 1992 y Donati, Andrade y Jubert 1992) se han explorado las ideas previas de los alumnos ingresantes a este centro, las cuales, en su mayoría, han corroborado hallazgos encontrados por otras investigaciones en la vasta bibliografía sobre ideas previas.

Las ideas y dificultades detectadas son:

Unidad I: materia

- la materia es de naturaleza contínua;

- la inexistencia de espacios vacíos en la materia;

- los gases no pesan;

- la masa de un cuerpo depende de su forma;

- la atribución de la masa de un átomo gramo a la masa de Ia partícula átomo. Igualmente con molécula gramo y masa de una molécula.

\section{Unidad 2: compuestos}

- el desconocimiento de los principales iones de uso común en química;

- la utilización parcial de métodos complejos memorizados para la obtención de compuestos, sobre todo aquéllos que emplean ecuaciones químicas.

\section{Unidad 3: soluciones}

- la generalización de la aditividad de los volúmenes de soluto y solvente;

- la idea que la concentración de una solución cambia con el volumen considerado de la misma;

- la utilización de la densidad de la solución como una concentración;

- las dificultades para plantear los cálculos básicos relacionados con la preparación de una solución de concentracion dada. 


\section{Unidad 4: reacciones químicas}

- Ia indiferenciación entre fenómenos físicos y quími$\cos$;

- la no conservación de los elementos en una reacción química;

- la interpretación confusa de la representación simbólica de las reacciones químicas.

\section{Unidad 5: estequiometría}

- la no conservación de las cantidades en una reacción química;

- la indiferenciación entre cantidades de combinación y cantidades asociadas a una situación experimental particular.

\section{Unidad 6: estequiometría con soluciones}

- la no individualización de las cantidades de los reactivos que participan en la reacción química al estar en solución;

- la no consideración de los excesos de reactivos como formando parte del conjunto obtenido luego de la reacción;

- la combinación de errores de las unidades anteriores.

\section{SECUENCIA DE ENSEÑANZA}

Para favorecer una participación activa de los estudiantes se puso en práctica un ciclo de aprendizaje (Karplus 1978) que consiste en una secuencia didáctica simple (motivación, introducción conceptual y aplicación). Además, se tuvieron en cuenta las formulaciones de Driver (1988) sobre una secuencia de enseñanza basada en el cambio conceptual y estrategias integradas como requisitos para el cambio conceptual (Pozo 1987).

En la primera etapa de motivación se fomenta que los alumnos hagan explícitas sus ideas previas. Se presentan contraejemplos y situaciones que las contradigan; se utilizan preguntas motivadoras y reflexivas, así como breves experiencias. El docente da espacio para que los alumnos anticipen y discutan entre ellos las cuestiones. Es importante dejar un tiempo de espera después de formular cada pregunta, para que todos razonen y elaboren sus hipótesis. Luego, cuando los alumnos formulan al grupo sus puntos de vista, el docente trata de confrontar respuestas contradictorias entre sí y fomenta la argumentación y discusión.

En la segunda etapa de introducción de conceptos se trabaja sobre la discusión anterior, buscando la construcción y presentación de un cuerpo de conceptos articulados entre sí. Se resaltan las ventajas de su uso y se muestra su utilidad en la explicación de nuevos fenóme- nos. Un recurso a utilizar, en esta etapa, son las redes conceptuales.

En la tercera etapa de aplicación se plantean situaciones y problemas que pretenden poner en juego los contenidos presentados. Se expresan no como ejercicios puramente deductivos o matemáticos sino fuertemente conceptuales en donde el alumno tenga fundamentalmente que pensar y tomar conciencia de las implicaciones de tos conceptos.

Se procuró que esta secuencia se vivenciara naturalmente y sin cortes entre sus fases.

\section{NÚCLEOS CONCEPTUALES}

Los núcleos conceptuales, especiaimente el referido a las conservaciones de propiedades no observables de la materia, constituyen ejes de articulación entre las unidades temáticas. La interpretación particular de la materia surge como un auxiliar explicativo imprescindible para la comprensión del núcleo anterior, y ambos constituyen requisitos cognitivos necesarios para la comprensión de otros conceptos químicos centrales del curso.

La mayor parte de las situaciones planteadas en la etapa de motivación fue tratada con ejemplos de conservaciones no visibles de la materia, como la conservación de la cantidad de partículas en una transformación de soluto al formar una solución; o la conservación del tipo de partículas, como por ejemplo la conservación de los elementos en una reacción química.

La adquisición de estos invariantes facilita el aprendizaje de procesos más complejos, como formar una solución a partir de otra solución (habilidad esencial de las químicas básicas) en la cual se debe mantener invariante la cantidad de partículas de soluto, que anteriormente se encontraba disuelta en cierta cantidad de solvente y que posteriormente estarán en otro volumen de solución, presentando una concentración distinta a la anterior. También otros procesos como dilución, evaporación y mezcla de soluciones se apoyan en estas habilidades de razonamiento.

Este tipo de conservaciones, que van más allá de la percepción inmediata, fue considerado por Inhelder y Piaget (1955) como uno de los ocho esquemas del pensamiento formal.

\section{INVESTIGACIÓN PREVIA}

Para mostrar el estado de conocimientos de los alumnos que realizan este curso preuniversitario, nos extenderemos sobre el diagnóstico realizado el año anterior antes de comenzar el curso respectivo.

Fueron planteadas situaciones referidas a conservaciones de propiedades de la materia y modelo corpuscular, en forma escrita, como resolución de ejemplos, a una 
menclatura inorgánica. Este autor, luego de un análisis de los textos de química general, en EEUU, Ilega a la conclusión de que éstos proponen mecanismos no sistemáticos y sujetos a Ia pura memorización, y encuentra que la mayoría de los profesores lo enseñan inadecuadamente o directamente evitan el tema.

Fue de utilidad para los alumnos el juego de los «naipes iónicos» (Cervelli et al. 1987), como una forma divertida de aplicar y reforzar los conocimientos. Consiste en una adaptación de la «escoba de 15», en donde, en este caso, en vez de formar la cantidad de 15 , se forma un compuesto químico, combinando iones positivos con negativos, con la condición đe que para «levantarlo" se debe nombrar el compuesto que se realiza. Este juego es una materialización operativa de las reglas dadas para la formulación de compuestos.

\section{RESULTADOS Y CONCLUSIONES FINALES}

En el examen final del curso se evaluó la adquisición de habilidades básicas para un exitoso desempeño posterior en las asignaturas de primer año de la universidad. Los ítems considerados fueron seis:

- utilización de unidades y cantidades de la materia (mol, átomo y molécula gramo, peso de un átomo y molécula, etc.);

- nombrar y clasificar compuestos químicos inorgáni$\cos$;

- calcular la concentración de una mezcla que resulta una solución saturada y la cantidad de reactivo precipitado;

- preparar una solución a partir de otra solución (volumétrica);

\section{REFERENCIAS BIBLIOGRÁFICAS}

ANDERSSON, B., 1990. Pupils'conceptions of matter and its transformations, Studies in Science Education, Vol. 18, pp. 53-85.

AUSUBEL, D.P., NOVAK, J.D. y HANESIAN, H., 1983. Psicologia educativa. (Educational Psycology, 1978). (Triłlas: Méjico).

CERVELLI, A.L. et al., 1987. Actividades para Química I. (Colihue: Buenos Aires).

DONATI, E., ANDRADE, G.J, y JUBERT, A., 1992. Algunos desconceptos en la enserianza de la estequiometría. Comunicación presentada en la VI Reunión Nacional de Educación en la Química. Tucumán. Argentina.
- relacionar estequiométricamente cantidades en una reacción química;

- determinar el reactivo en exceso, de una reacción entre dos solutos en una mezcla de soluciones, y calcular la concentración resultante.

Como se puede apreciar, se exigieron, de los alumnos, capacidades cognitivas no sencillas para estudiantes preuniversitarios que no cuentan con una base sólida đe conocimientos en química y que tampoco han tenido oportunidades anteriores para revisar y cuestionar en profundidad sus concepciones sobre estos temas. Nuestra impresión sobre los aprendizajes de química que poseen los alumnos que egresan de la escuela secundaria es que son nulos, confusos o de poca utilidad, dado el fuerte predominio memorístico sobre el conceptual.

Los resultados obtenidos (el $62 \%$ de los alumnos superó el $60 \%$ mínimo requerido) nos muestran una mejora con respecto a años anteriores (resultados menores al $50 \%$ de aprobación), que lo atribuímos a la adecuada selección de contenidos, organización del material y secuencia apropiada de enseñanza; en síntesis a un abordaje no ingenuo del problema, que procuró partir de los conocimientos previos que poseen los estudiantes. Por otra parte, a lo largo del desarrollo del curso, se observaron en los alumnos el logro de actitudes positivas como ser una mayor motivación y un mayor grado de independencia en la resolución de problemas.

A lo targo de estas páginas se intentó mostrar una experiencia en la cual, aunque sea muy común atribuir las deficiencias de los alumnos a la escuela secundaria ( $y$ la universidad ocuparse solamente de los que logran «espontáneamente» ponerse al día), es posible que se brinde un espacio de revisión y aprendizaje conceptual que permita a muchos estudiantes tener un mejor desempeño en sus estudios y quizás superar, en alguna medida, el elevado índice de fracasos que se observa en el primer año de la universidad argentina.

DRIVER, R., 1986. Psicología cognoscitiva y esquemas conceptuales de los alumnos, Enseñanza de la Ciencias, Vol. $4(1)$, pp. 3-15.

DRIVER, R., 1988. Un enfoque constructivista para el desarrollo del currículo de ciencias, Enseñanza de las Ciencias, Vol. $6(2)$, pp. 109-120.

EBENEZER, J.V., 1992. Making chemistry learning more meaningful, Journal of Chemical Education, Vol. 69(6), pp. 464-467.

FURIÓ MAS, C. y GIL PÉREZ, D., 1988. Aportaciones de la investigación didáctica al proceso de enseñanza-aprendizaje de la química. Comunicación presentada en la $P R A I M E Q-1$. San Luis. Argentina. 
INHELDER, B. y PIAGET, J., 1972. De la lógica del niño a la lógica del adolescente. (De la logique de l'enfant a la logique de I'adolescent, 1955). (Paidós: Buenos Aires)

KARPLUS, R., 1978. La enseñanza de las ciencias a los alumnos jóvenes, Perspectivas, Vol. 8(1), pp. 49-59.

LIND, G., 1992. Teaching Inorganic Nomenclature, Journal of Chemical Education, Vol. 69(8), pp. 613-614.

LLORENS MOLINA, J.A, 1988. La concepción corpuscular de la materia. Obstáculos epistemológicos y problemas de aprendizaje, Investigación en la Escuela, Vol.4, pp. 33-48.

NAKHLEH, M.B., 1992. Why somestudents don't leam chemistry, Journal of Chemical Education, Vol. 69(3), pp. 191-196.

NOVAK, J.D y GOWIN, D.B., 1988. Aprendiendo a aprender. (Learning how to learn, 1984). (Martínez Roca: Barcelona).

NURRENBERN, S.C. y PICKERING, M., 1987.Concept learning versus problem solving, Journal of Chemical Education, Vol. 64(6), pp. 508-510.

OÑORBE DE TORRE, A. y SÁNCHEZ JIMÉNEZ, J.M., 1992. La masa no se crea ni se destruye. ¿Estáis seguros?, Enseñanza de las Ciencias, 10(2), pp. 165-171.
PIAGET, J. e INHELDER, B., 1971. El desarrollo de las cantidades en el niño. (Le developpement des quantités physiques chez l'enfant, 1941). (Nova Terra: Barcelona).

POZO, J.I., 1987. Aprendizaje de la ciencia y pensamiento causal. (Visor: Madrid).

POZO, J.I., CRESPO, M., SANZ, A. y LIMONN, M., 1992. La estructura de los conocimientos en química: una proptuesta de núcleos conceptuales, Investigación en la Escuela, Vol. 18 , pp. 23-39.

RAVIOLO, A., 1992. Curso de nivelación en química al ingreso a la universidad: investigación didáctica sobre las ideas previas de los alumnos. Comunicación presentada en la VI Reunión Nacional de Educación en la Química. Tucumán. Argentina.

SHAYER, M. y ADEY, P., 1984. La ciencia de enseñar ciencias. (Towards a science of science teaching, 1981). (Narcea: Madrid).

STAVY, R., 1991. Using analogy to overcome misconceptions about conservation of matter, Journal of Research in Science Teaching, Vol. 28(4), pp. 305-313.

\section{ANEXO}

Figura 1

Red conceptual unidad 1: materia.




Figura 2

Red conceptual unidad 3: soluciones.

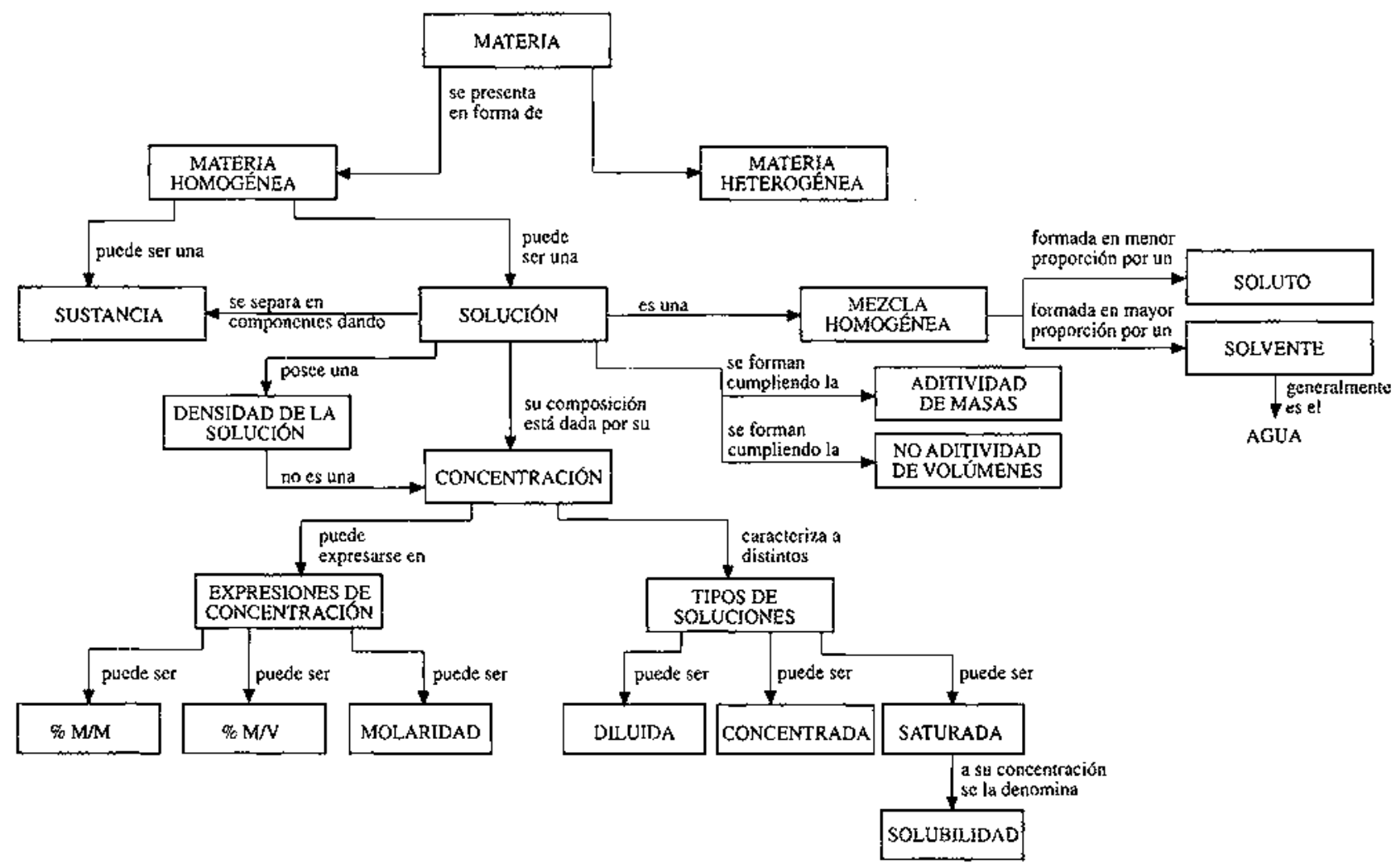

